\title{
Comparação da performance funcional do membro inferior entre jovens futebolistas e jovens não treinados
}

\author{
Comparison of lower limb functional performance between young \\ soccer players and untrained young men
}

\author{
Telmo Silva $^{[a]}$, Fernando Ribeiro ${ }^{[\mathrm{b}]}$, João Venâncio ${ }^{[\mathrm{c}]}$
}

[a] Fisioterapeuta, Prática Privada de Fisioterapia, Licenciado pela Escola Superior de Saúde do Vale do Sousa, Gandra, Portugal, e-mail: telmomarcos@gmail.com

[b] Fisioterapeuta, Mestre em Ciências do Desporto, Departamento de Fisioterapia, Escola Superior de Saúde do Vale do Sousa, Gandra, Portugal e Centro de Investigação em Atividade Física, Saúde e Lazer, Faculdade de Desporto da Universidade do Porto, Porto, Portugal, e-mail: fernando.silva.ribeiro@gmail.com

[c] Fisioterapeuta, Mestre em Ciências do Desporto. Departamento de Fisioterapia, Escola Superior de Saúde do Vale do Sousa, Gandra, Portugal e Centro de Investigação em Atividade Física, Saúde e Lazer, Faculdade de Desporto da Universidade do Porto, Porto, Portugal, e-mail:venancio.joao@gmail.com

\section{Resumo}

Introdução: Em atletas, a utilização de testes de performance funcional permite avaliar com maior validade ecológica possíveis alterações do membro inferior observadas na prática desportiva. Objetivo: O objetivo do presente estudo consistiu na comparação da performance funcional do membro inferior entre jovens futebolistas e jovens não treinados. Metodologia: A amostra foi constituída por 39 jovens do sexo masculino, divididos em dois grupos: um grupo composto por 19 futebolistas (idade $=16,8 \pm 0,83$ anos; peso $=69,8 \pm 6,2 \mathrm{~kg}$; altura $=176,6 \pm 0,5 \mathrm{~cm}$ ) e outro composto por 20 sujeitos não treinados (idade $=16,6 \pm 0,8$ anos; peso $=66,3 \pm 11,0 \mathrm{~kg}$; altura $=$ $172,0 \pm 0,8 \mathrm{~cm}$ ). Para avaliação da performance funcional do membro inferior foram utilizados três testes: o Single-Leg Hop Test, o Quadrant Jump Test e o Hexagon Test. Resultados: Os jovens futebolistas obtiveram melhor performance do que os jovens não treinados em ambos os membros inferiores no Single-Leg Hop Test (membro inferior direito: $124 \pm 62 \mathrm{~cm}$, membro inferior esquerdo: $127 \pm 68 \mathrm{~cm}$ versus membro inferior direito: $99 \pm 10 \mathrm{~cm}$, membro inferior esquerdo: $97 \pm 12 \mathrm{~cm}$; $\mathrm{p}<0.001$ ), em ambas as direções, na execução do Hexagon Test (sentido dos ponteiros do relógio: $5,1 \pm 0,6 \mathrm{~s}$, sentido inverso: 4,9 $\pm 0,5 \mathrm{~s}$ versus sentido dos ponteiros do relógio: $6,0 \pm 0,4 \mathrm{~s}$, sentido inverso: $5,7 \pm 0,4 \mathrm{~s} ; \mathrm{p}<0.001)$ e no Quadrant Jump Test $(32,4 \pm 2,5$ pontos versus $28,2 \pm 2,5$ pontos, $\mathrm{p}<0.001)$. Dentro de cada grupo não foi encontrada diferença entre os dois membros inferiores no Single-Leg Hop Test, nem entre as duas direções do Hexagon Test. Conclusões: Os resultados do presente estudo indicam que jovens futebolistas apresentam melhor performance funcional do membro inferior do que jovens não treinados.

Palavras-chave: Futebol. Lesões. Membro inferior. Performance funcional. 


\begin{abstract}
Introduction: In athletes, functional performance tests are useful tolls to identify alterations in the lower limb performance, as they have more ecological validity than lab tests. Objective: This study aimed to compare the lower limb functional performance between young footballers and non-trained young subjects. Methods: Thirty-nine young male subjects were recruited and divided into two groups: one group was composed by 19 footballers (aged $=16.8 \pm$ 0.83 years; weight $=69.8 \pm 6.2 \mathrm{~kg}$; height $=176.6 \pm 0.5 \mathrm{~cm})$ and the other was composed by 20 non-trained subjects (aged $=16.6 \pm 0.8$ years; weight $=66.3 \pm 11.0 \mathrm{~kg}$; height $=$ $172.0 \pm 0.8 \mathrm{~cm})$. The Single-Leg Hop Test, the Quadrant Jump Test, and the Hexagon Test were used to assess the functional performance of the lower limb. Results: The young footballers performed better than the non-trained subjects in the Single-Leg Hop Test, in both lower limbs (right lower limb: $124 \pm 62 \mathrm{~cm}$, left lower limb: $127 \pm 68 \mathrm{~cm}$ versus right lower limb: $99 \pm 10 \mathrm{~cm}$, left lower limb: $97 \pm 12 \mathrm{~cm} ; p<0.001)$, in both directions of the Hexagon Test (in the direction of the clock pointers: $5.1 \pm 0.6 \mathrm{~s}$, in the opposite direction of the clock pointers: $4.9 \pm 0.5$ s versus $6.0 \pm 0.4 s, 5.7 \pm 0.4$ s, respectively; $p<0.001$ ), and in the Quadrant Jump Test (32.4 \pm 2.5 points versus $28.2 \pm 2.5$ points, $p<0.001)$. Within each group, no differences were found between both lower limbs in the Single-Leg Hop Test, neither in both directions of the Hexagon Test. Conclusions: It can be concluded that young football players have better functional performance of the lower limb than non-trained young subjects.
\end{abstract}

Keywords: Football. Injuries. Lower limb. Functional performance.

\title{
Introdução
}

O futebol é a modalidade desportiva mais praticada no mundo, caracterizando-se por apresentar grande contato físico, movimentos curtos e rápidos, tais como acelerações e desacelerações, mudanças de direcção, saltos e rotações $(1,2)$. No futebolista, as articulações do membro inferior, nomeadamente do joelho e tornozelo, são as que apresentam maior incidência de lesões (3-5). Dos vários fatores que contribuem para a elevada incidência de lesões no membro inferior, destacamos os défices proprioceptivos, de coordenação motora, de capacidade neuromuscular e, consequentemente, de desempenho funcional (6-9). Esses défices são observados em situações de lesão e fadiga e passíveis de ser mensurados por meio de testes de performance funcional, os quais são compostos por um conjunto de medidas dinâmicas que permitem avaliar de forma combinada um conjunto de variáveis nas quais estão incluídas a força muscular, a coordenação neuromuscular, o equilíbrio, a agilidade e a propriocepção. Clinicamente, os testes de performance funcional poderão ser utilizados para avaliar o risco de lesão e nas etapas finais de reabilitação como critério para determinar o retorno à atividade $(10,11)$.

Em atletas, a utilização de testes funcionais, nos quais a exigência se aproxima ou reproduz as exigências da atividade desportiva, permite avaliar mais fielmente e com maior validade ecológica possíveis alterações da performance funcional observadas em contextos formais de atividade desportiva. A literatura fornece poucos estudos comparando a performance funcional de jovens futebolistas e jovens não praticantes de exercício físico. Desta forma, constituiu-se como objetivo deste estudo a comparação da performance funcional do membro inferior entre jovens futebolistas e jovens não treinados. 


\section{Material e métodos}

\section{Participantes}

A amostra, de conveniência, deste estudo transversal foi constituída por 39 indivíduos voluntários do sexo masculino, divididos em dois grupos. O grupo de futebolistas foi composto por 19 sujeitos (idade $=16,8 \pm 0,83$ anos; peso $=69,8 \pm 6,2 \mathrm{~kg}$; altura $=176,6 \pm 0,5 \mathrm{~cm}$ ), sendo o grupo de não treinados constituído por 20 sujeitos (idade $=16,6 \pm 0,8$ anos; peso $=66,3 \pm 11,0 \mathrm{~kg}$; altura $=172,0$ $\pm 0,8 \mathrm{~cm}$ ). Foram excluídos do estudo todos os participantes que apresentassem: (i) qualquer tipo de lesão atual, ou em fase de reabilitação, nos membros inferiores; (ii) histórico de patologia ou cirurgia em membros inferiores; (iii) qualquer patologia que pudesse afetar a performance; (iv) estivessem tomando medicação que afetasse o equilíbrio, a coordenação ou a memória. Foi definido como critério de inclusão no grupo de futebolistas uma frequência de treino semanal mínima de 8 horas. Todos os sujeitos deram o seu consentimento informado por escrito e todos os procedimentos foram efetuados segundo as recomendações da Declaração de Helsínquia.

\section{Testes de avaliação da performance funcional do membro inferior}

Para avaliação da performance funcional do membro inferior foram utilizados três testes: o Single-Leg Hop Test, o Quadrant Jump Test e o Hexagon Test. Previamente à execução de cada um dos testes, cada um dos sujeitos realizou um ensaio de familiarização.

No Single-Leg Hop Test, os sujeitos foram instruídos a saltar o mais longe possível em apoio unipodálico com a mesma perna (Figura 1). O teste foi realizado três vezes para cada membro, alternando entre direito e esquerdo. A distância de cada salto foi medida em centímetros a partir da posição inicial do teste até o ponto de apoio. Foi considerado como valor do teste a melhor marca alcançada nas três tentativas. Utilizou-se neste estudo a versão modificada do Single-Leg Hop Test, que permite movimentar os braços livremente durante o salto, visando maior funcionalidade na sua execução (12).

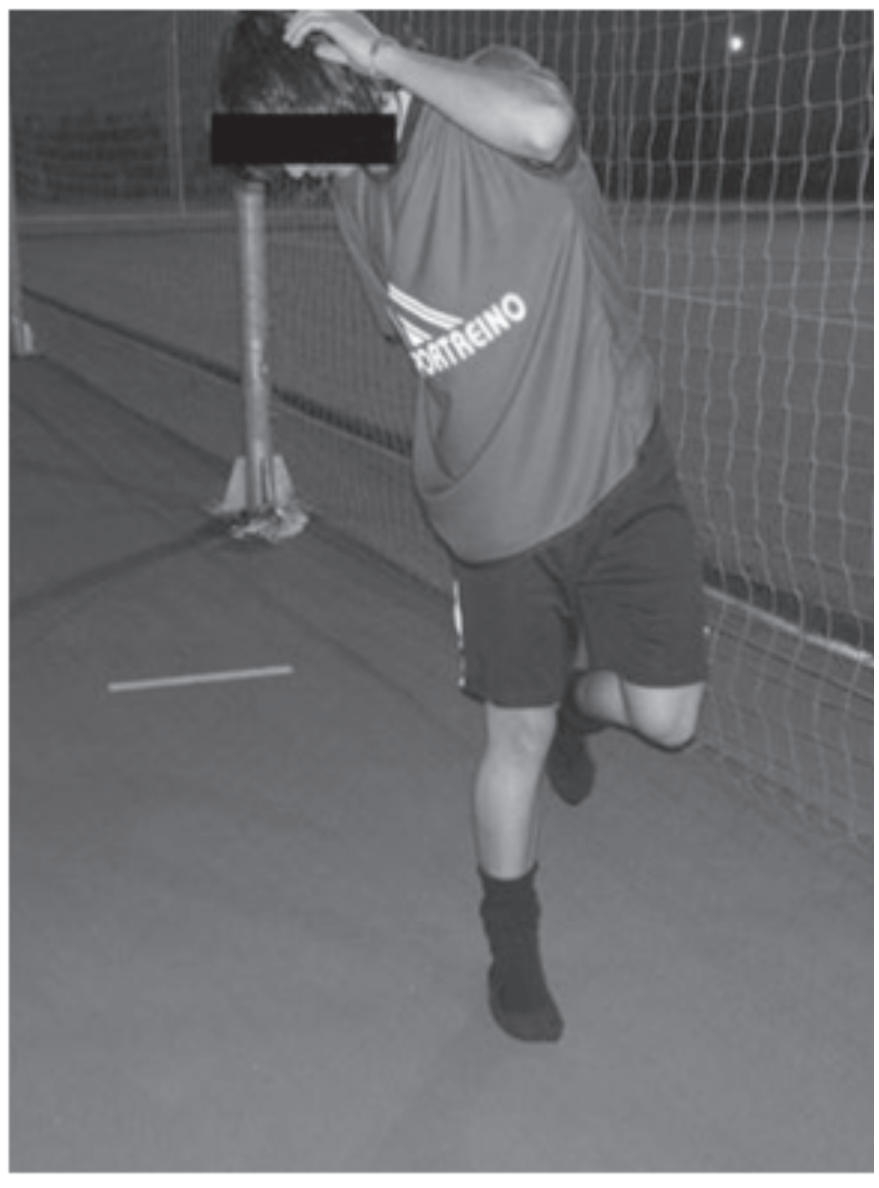

Figura 1 - Single-leg hop test 
O Quadrant Jump Test (Figura 2) foi originalmente descrito por Johnson e Nelson em 1986 (13). É desenhada uma cruz perpendicular no chão, com tape. O quadrante deve ter um metro de comprimento por um metro de largura. É colocada uma linha de "início" no primeiro quadrante e os restantes são numerados na sequência 1, 2, 3, 4. O indivíduo salta com os pés juntos sucessivamente na sequência $1,2,3,4,1$, 2, 3, 4 o mais rápido possível, durante 10 segundos. É atribuído 1 ponto sempre que o indivíduo salta corretamente com ambos os pés no quadrante correto, sendo atribuída uma penalização de 0,5 pontos sempre que o indivíduo pisa a linha ou salta no quadrante errado. A pontuação final representa o número de vezes que o sujeito salta corretamente no quadrante, descontando 0,5 pontos a cada vez que salta na linha ou no quadrante errado. Foram efetuados 3 ensaios, sendo considerado, para efeitos de classificação, a média dos dois melhores ensaios.

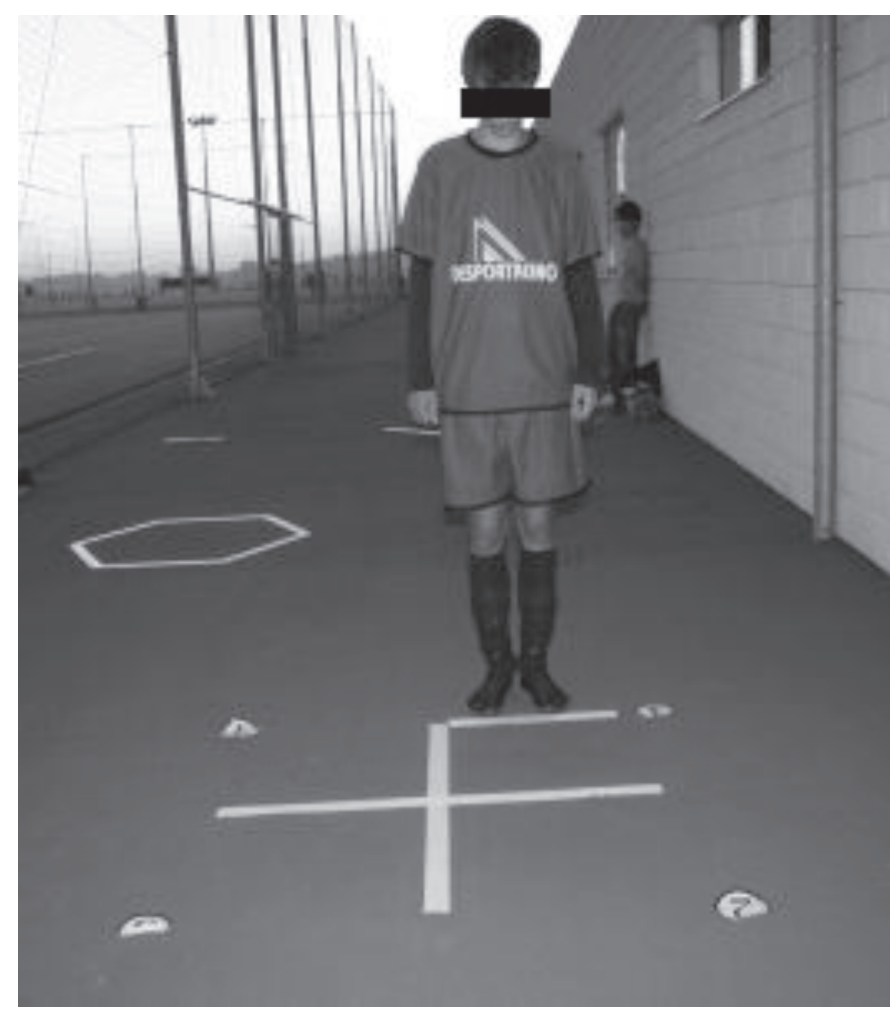

Figura 2 - Quadrant jump test

O Hexagon Test foi executado segundo o protocolo estabelecido por Baechle e Earle (14). Desenha-se um hexágono no chão com $60,5 \mathrm{~cm}$ de lado e um ângulo externo de $120^{\circ}$ (Figura 3). O indivíduo coloca-se no centro do hexágono com os pés juntos, sobre o comando "ir", salta para a frente e volta ao centro do hexágono, repetindo a tarefa em todas as direções do hexágono no sentido dos ponteiros do relógio, seguidamente executa a mesma tarefa no sentido inverso aos ponteiros do relógio. O teste é reiniciado sempre que o indivíduo salta na linha errada ou avança um lado do hexágono. Foram realizados 3 ensaios em cada sentido. O tempo de execução foi registado. Para análise, considerouse a média dos dois melhores tempos em cada um dos sentidos.

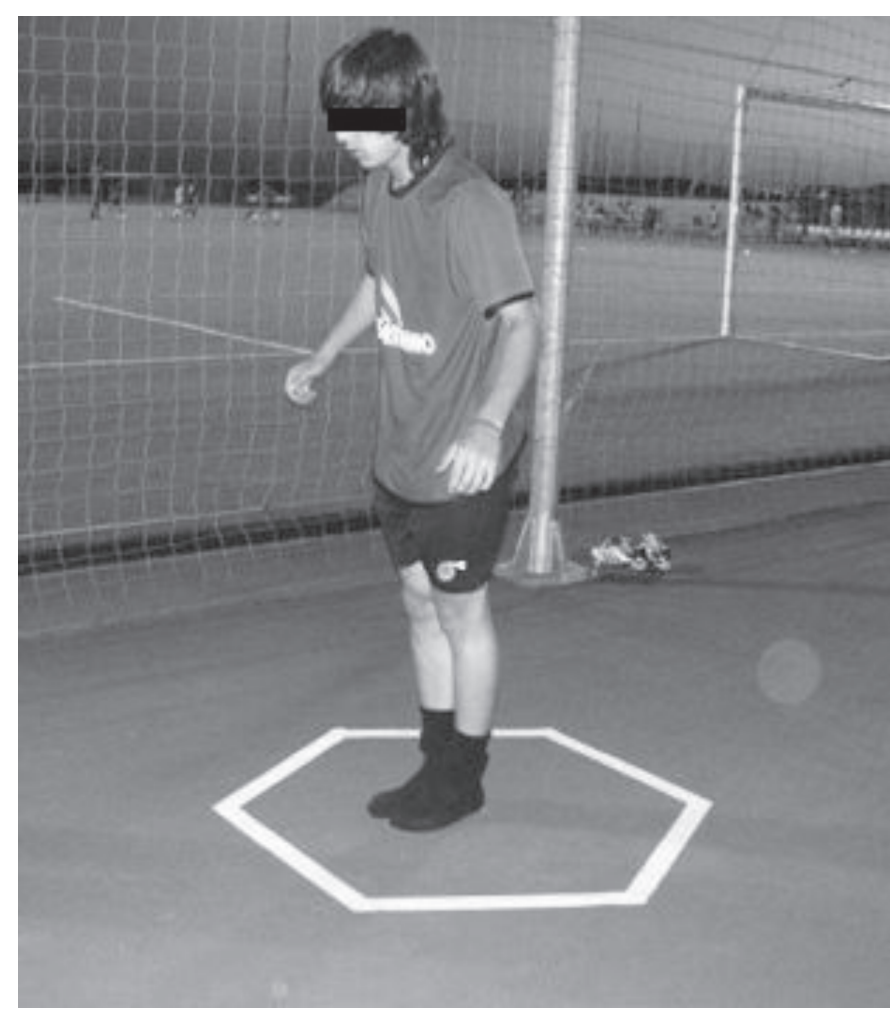

Figura 3 - Hexagon test 


\section{Análise estatística}

O tratamento estatístico foi realizado utilizando o aplicativo Statistical Package for Social Sciences versão 16.0 (SPSS Inc., Chicago, IL). Foi utilizada estatística descritiva para calcular a média e o desvio-padrão. As médias dos dois grupos foram comparadas com recurso ao Teste t de Student para amostras independentes. Utilizou-se o Teste $t$ de Student nas amostras emparelhadas para comparar, dentro de cada grupo, as médias entre os dois membros no Single-Leg Hop Test e entre as duas direcções no Hexagon Test. O nível de significância foi estabelecido em 5\%.

\section{Resultados}

Todos os sujeitos que participaram do estudo completaram com sucesso as tarefas que lhes foram determinadas. Os jovens futebolistas apresentaram melhor performance em todos os testes funcionais efetuados (Tabela 1). Observaram-se diferenças significativas entre grupos em ambos os membros inferiores no Single-Leg Hop Test e em ambas as direcções na execução do Hexagon Test, com o grupo de futebolistas a realizar melhor performance com ambos os membros inferiores e em ambas as direcções (sentido/sentido inverso dos ponteiros do relógio). Contudo, dentro de cada grupo não se verificaram diferenças entre membros inferiores no Single-Leg Hop Test, nem entre direcções no Hexagon Test.

Tabela 1 - Comparação dos valores médios nos testes de performance entre o grupo de futebolistas e o grupo de sujeitos não treinados

\begin{tabular}{lccccc}
\hline Single-leg hop test $(\mathrm{cm})$ & Hexagon test (s) & $\begin{array}{c}\text { Quadrant jump } \\
\text { (pontos) }\end{array}$ \\
\hline & M.I.D. & M.I.E. & S.P.R & S.I.P.R & \\
Futebolistas & $124 \pm 62$ & $127 \pm 68$ & $5.1 \pm 0.6$ & $4.9 \pm 0.5$ & $32.4 \pm 2.5$ \\
Não treinados & $99 \pm 10^{*}$ & $97 \pm 12^{*}$ & $6.0 \pm 0.4^{*}$ & $5.7 \pm 0.4^{*}$ & $28.2 \pm 2.5^{*}$ \\
\hline
\end{tabular}

\section{Discussão}

O presente estudo teve por objectivo a comparação da performance funcional do membro inferior entre jovens futebolistas e jovens não treinados. Os resultados indicam que a performance funcional do membro inferior avaliada através do Quadrant Jump Test, Single-Leg Hop Test e Hexagon Test é significantemente melhor em jovens futebolistas do que em jovens não treinados, podendo estes, desta forma, apresentar maior protecção para lesões no contexto da sua prática desportiva.

A medição de resultados em fisioterapia desportiva é fundamental para identificar atletas em risco de lesão e para prevenir o risco de recidiva no retorno à competição. A criação de bases de dados com os valores da performance funcional dos atletas no início da época revelam-se de extrema importância para o processo de tomada de decisão do fisioterapeuta sobre o momento no qual o atleta em processo de recuperação já se encontra apto para retomar a competição. Esse processo reveste de grande complexidade, porque em inúmeros casos, apesar dos atletas se apresentarem assintomáticos, os seus parâmetros de força muscular, coordenação neuromuscular, equilíbrio, agilidade e propriocepção ainda não se encontram nos níveis que este detinha no período pré-lesão. Nesse sentido, os dados objetivos fornecidos pelos testes de performance funcional, testes com boa relação custo/benefício, de 
fácil aplicabilidade fora do ambiente laboratorial e que se aproximam do contexto formal do futebol, podem ser de extrema importância. Os resultados do presente estudo indicam que estes testes permitem distinguir entre jovens futebolistas e jovens não treinados, apresentando, tal como seria expectável, estes últimos pior performance.

Os atletas possuem características morfológicas e fisiológicas específicas e estão expostos a um conjunto de exigências físicas inerentes às tarefas motoras presentes em cada modalidade (15-17). Esses dois fatores podem explicar, pelo menos parcialmente, as diferenças na performance funcional do membro inferior encontradas no presente estudo. As diferenças encontradas no presente estudo, poderão ser explicadas não só pelo expectável aumento da força muscular induzido pela prática de futebol, mas também pela melhor acuidade proprioceptiva e, concomitantemente, melhor controle motor e estabilidade postural associado à prática regular e repetitiva dos gestos desportivos. De fato, contrariamente à fadiga muscular (18-21), a prática regular de exercício físico melhora a propriocepção articular $(22,23)$, por meio de mecanismos relacionados com a sua componente periférica (nomeadamente promovendo adaptações morfológicas no fuso neuromuscular) e com a sua componente central (modulando o ganho do fuso neuromuscular e induzindo adaptações plásticas no Sistema Nervoso Central) [para revisão ver (24-26)].

Relativamente ao Single-Leg Hop Test, a sua performance está intimamente relacionada com a capacidade de gerar força concêntrica na fase de descolagem e excêntrica na fase de recepção, principalmente pelos músculos extensores do joelho $(27,28)$. Num estudo recente em jovens futebolistas, Hamilton e colaboradores (29) confirmaram a importância da força e da potência muscular para a performance nos testes de distância horizontal. Os autores encontraram uma relação positiva entre a performance funcional e a potência e força muscular do quadricípite e isquiotibiais. Da mesma forma, nossos resultados no Hexagon Test e no Quadrant Jump Test vão de encontro aos resultados de estudos prévios, nos quais se verificou jovens atletas apresentarem melhor performance do que jovens não atletas $(15,30)$.

\section{Conclusão}

Os resultados do presente estudo permitem concluir que jovens futebolistas apresentam melhor performance funcional do membro inferior do que jovens não treinados. A melhoria do desempenho nos testes de performance funcional parece estar relacionada com as adaptações crônicas induzidas pelo exercício físico na capacidade neuromuscular e proprioceptiva. No entanto, investigações futuras são importantes nesta área para a exata compreensão dos mecanismos subjacentes ao aumento da performance funcional dos membros inferiores induzido pelo prática regular de exercício físico. Futuros estudos deverão também avaliar possíveis diferenças da performance funcional do membro inferior entre sexos, bem como em diferenças coortes de idades, e entre modalidades desportivas com diferente exigência competitiva. Adicionalmente, a contribuição de melhores resultados nos testes de performance funcional do membro inferior para menor incidência de lesões no decorrer de uma época também deverá ser alvo de investigação futura.

\section{Referências}

1. Lennox JW, Rayfield J, Steffen B. Soccer Skills \& Drills. Champaign: Human Kinetics; 2006.

2. Hargreaves A, Bate R. Skills \& Strategies for coaching soccer. Champaign: Human Kinetics; 1990.

3. Junge A, Dvorak J, Graf-Baumann T, Peterson L. Football injuries during FIFA tournaments and the Olympic Games, 1998-2001: development and implementation of an injury-reporting system. Am J Sports Med. 2004;32(1 Suppl):80S-9S. 
4. Junge A, Dvorak J, Graf-Baumann T. Football injuries during the World Cup 2002. Am J Sports Med. 2004;32(1 Suppl):23S-7S.

5. Junge A, Dvorak J. Injuries in female football players in top-level international tournaments. Br J Sports Med. 2007;41(Suppl 1):i3-7.

6. Ireland ML. Anterior cruciate ligament injury in female athletes: epidemiology.J Athl Train. 1999;34(2):150-154.

7. Hrysomallis C. Relationship between balance ability, training and sports injury risk. Sports Med. 2007;37(6):547-56.

8. Junge A, Dvorak J. Soccer injuries: a review on incidence and prevention. Sports Med. 2004;34(13):929-38.

9. Alentorn-Geli E, Myer GD, Silvers HJ, Samitier G, Romero D, Lazaro-Haro C, Cugat R. Prevention of non-contact anterior cruciate ligament injuries in soccer players. Part 1: Mechanisms of injury and underlying risk factors. Knee Surg Sports Traumatol Arthrosc. 2009;17(7):705-29.

10. Docherty CL, Arnold BL, Gansneder BM, Hurwitz S, Gieck J. Functional-performance deficits in volunteers with functional ankle instability. J Athl Train. 2005;40(1):30-4.

11. Clark NC. Functional performance testing following knee ligament injury. Phys Ther Sport. 2001;2:91-105.

12. Zatterstrom R, Friden T, Lindstrand A, Moritz U. Rehabilitation following acute anterior cruciate ligament injuries-a 12-month follow-up of a randomized clinical trial. Scand J Med Sci Sports. 2000;10(3):156-63.

13. Johnson BL, Nelson JK. Practical measurements for evaluation in physical education. Minnessota: Burgess Publishing; 1986.

14. Baechle T, Earle R. Essentials of strength and conditioning. Windsor, Ontario: Human Kinetics; 2000.

15. Eisenmann JC, Malina RM. Age- and sex-associated variation in neuromuscular capacities of adolescent distance runners. J Sports Sci. 2003;21(7):551-7.

16. Reilly T, Bangsbo J, Franks A. Anthropometric and physiological predispositions for elite soccer. J Sports Sci. 2000;18(9):669-83.

17. Wong del P, Wong SH. Physiological profile of Asian elite youth soccer players. J Strength Cond Res. 2009;23(5):1383-90.

18. Ribeiro F, Oliveira J. Efeito da fadiga muscular local na propriocepção do joelho. Fisioter Mov. 2008;21(2):71-83.

19. Ribeiro F, Mota J, Oliveira J. Effect of exercise-induced fatigue on position sense of the knee in the elderly. Eur J Appl Physiol. 2007;99(4):379-85.

20. Miura K, Ishibashi Y, Tsuda E, Okamura Y, Otsuka H, Toh S. The effect of local and general fatigue on knee proprioception. Arthroscopy. 2004;20(4):414-8.

21. Forestier N, Teasdale N, Nougier V. Alteration of the position sense at the ankle induced by muscular fatigue in humans. Med Sci Sports Exerc. 2002;34(1):117-22.

22. Tsang WW, Hui-Chan CW. Effects of tai chi on joint proprioception and stability limits in elderly subjects. Med Sci Sports Exerc. 2003;35(12):1962-71.

23. Petrella RJ, Lattanzio PJ, Nelson MG. Effect of age and activity on knee joint proprioception. Am J Phys Med Rehabil. 1997;76(3):235-41.

24. Ribeiro F, Oliveira J. Aging effects on joint proprioception: the role of physical activity in proprioception preservation. Eur Rev Aging Phys Act. 2007;4:71-6. 
25. Goble DJ, Coxon JP, Wenderoth N, Van Impe A, Swinnen SP. Proprioceptive sensibility in the elderly: degeneration, functional consequences and plastic-adaptive processes. Neurosci Biobehav Rev. 2009;33(3):271-8.

26. Ashton-Miller JA, Wojtys EM, Huston LJ, Fry-Welch D. Can proprioception really be improved by exercises? Knee Surg Sports Traumatol Arthrosc. 2001;9(3):128-36.

27. Augustsson J, Thomee R, Linden C, Folkesson M, Tranberg R, Karlsson J. Single-leg hop testing following fatiguing exercise: reliability and biomechanical analysis. Scand J Med Sci Sports. 2006;16(2):111-20.

28. Orishimo KF, Kremenic IJ. Effect of fatigue on single-leg hop landing biomechanics. J Appl Biomech. 2006;22(4):245-54.

29. Hamilton RT, Shultz SJ, Schmitz RJ, Perrin DH. Triple-hop distance as a valid predictor of lower limb strength and power. J Athl Train. 2008;43(2):144-51.

30. Ortiz A, Olson SL, Roddey TS, Morales J. Reliability of selected physical performance tests in young adult women. J Strength Cond Res. 2005;19(1):39-44.

Recebido: 20/02/2009

Received: 02/20/2009

Aprovado: 13/10/2009

Approved: 10/13/2009 